\title{
Improved cuckoo search with particle swarm optimization for classification of compressed images
}

\author{
VAMSIDHAR ENIREDDY ${ }^{1, *}$ and REDDI KIRAN KUMAR ${ }^{2}$ \\ ${ }^{1}$ Department of CSE, JNT University Kakinada, Kakinada 533003, \\ Andhra Pradesh, India \\ ${ }^{2}$ Department of Computer Science, Krishna University, Machilipatnam 521001, \\ Andhra Pradesh, India \\ e-mail: enireddy.vamsidhar@gmail.com; kirankreddi@gmail.com
}

\begin{abstract}
The need for a general purpose Content Based Image Retrieval (CBIR) system for huge image databases has attracted information-technology researchers and institutions for CBIR techniques development. These techniques include image feature extraction, segmentation, feature mapping, representation, semantics, indexing and storage, image similarity-distance measurement and retrieval making CBIR system development a challenge. Since medical images are large in size running to megabits of data they are compressed to reduce their size for storage and transmission. This paper investigates medical image retrieval problem for compressed images. An improved image classification algorithm for CBIR is proposed. In the proposed method, RAW images are compressed using Haar wavelet. Features are extracted using Gabor filter and Sobel edge detector. The extracted features are classified using Partial Recurrent Neural Network (PRNN). Since training parameters in Neural Network are NP hard, a hybrid Particle Swarm Optimization (PSO) - Cuckoo Search algorithm (CS) is proposed to optimize the learning rate of the neural network.
\end{abstract}

Keywords. Content based image retrieval (CBIR); image compression; partial recurrent neural network (PRNN); particle swarm optimization (PSO); HAAR wavelet; Cuckoo Search (CS).

\section{Introduction}

In the medical field, CBIR's (Smeulders et al 2000) aim is to help radiologists to retrieve similar featured images leading to diagnosis similar to the input image. This differs from other fields where the aim is finding nearest image from same category images. CBIR based on color features is the heart of image database systems. Color descriptors are important features in image retrieval and analysis. Direct histogram comparison is a common technique to measure color similarity (Virk \& Maini 2011) due to its compact representation and low complexity. Shape features are

${ }^{*}$ For correspondence 
an effective method to index images, as many images provide limited texture information for anatomy of interest. Shape representation should be invariant to translation, rotation, and scaling in image retrieval systems.

Automatic medical image classification (Haralick et al 1973) assigns a medical image to a class among many images categories. Its task in CBIR is complicated by computational complexity. Many medical image retrieval systems which are available classify image according to image modalities, orientations and body part/disease. Medical images categorization means, selecting appropriate class for a given image from a set of pre-defined categories. Medical images classification modalities accuracy depends on image extracted feature, selected features classifier type. Classification accuracy is increased through the use of appropriate features combination with a specific classifier (Rajaei \& Rangarajan 2011).

CBIR attempts to bridge this gap with techniques in two categories: short-term learning attempts to learn which images are relevant to user's query over a single query, while long-term learning tries to learn intra-image connections over many queries. Both techniques are effective, and usually both are combined. In relevance feedback, labeling is used to give feedback as either relevant or irrelevant to query image and thus enables learning semantic information in images (Barrett 2007). While humans can match similar images or objects, machine vision research is still away from similar performance for computers. Currently, many retrieval approaches are based on low-level features such as color, texture, and shape features, leaving a 'semantic gap' to the high-level understanding of users (Schaefer 2010).

Image compression is a data compression application that encodes original image with few bits. Image compression's objective is reducing image redundancy and storing or transmitting data efficiently. The goal of such systems is to reduce storage quantity and displaying decoded image in the monitor which is as similar to the original image as much as possible (Wei 2008).

Recurrent Neural Networks (RNNs) have been developed to extend neural networks to sequential data. Due to their recurrent connections, RNNs use previous context (Graves et al 2007) and can adapt to compressed or stretched input patterns by varying change rate of internal state and thus are robust to temporal warping than other models. RNNs are effective for one dimensional sequence learning tasks, like speech/online handwriting recognition. RNNs permit self-connections, enabling them to gain knowledge of past events thereby permitting them to be flexible on temporal data. RNN is classified in two categories: partially and fully recurrent networks. Figure 1 shows a sample RNN.

RNN optimization has a major role in generalization performance. Regularization methods for network optimization include early stopping and weight decay (Vadapalli et al 2010). Early stopping in optimization ensures that training data is divided into training and validation sets. When training network using new training sets, it is also tested for validation error on the validation set. The network with lowest validation error is used for testing. But, early stopping suffers from over-fitting data where generalization performance is good for one set but fails in another.

In this paper, a methodology for the image retrieval of compressed medical images is proposed. An image classification based on partial recurrent neural network is proposed. In the proposed method, the images are compressed using Haar wavelet. Gabor filter and Sobel edge detector are used for feature extraction. The extracted features are classified using Partial Recurrent Neural Network (PRNN). A novel Particle Swarm Optimization (PSO) - Cuckoo Search algorithm (CS) is used for optimization of the learning rate of the neural network. The rest of the paper is organized as follows: Section 2 reviews some of the work available in the literature, section 3 details the materials and methods used in this investigation, section 4 reports the experimental results and section 5 concludes the paper. 


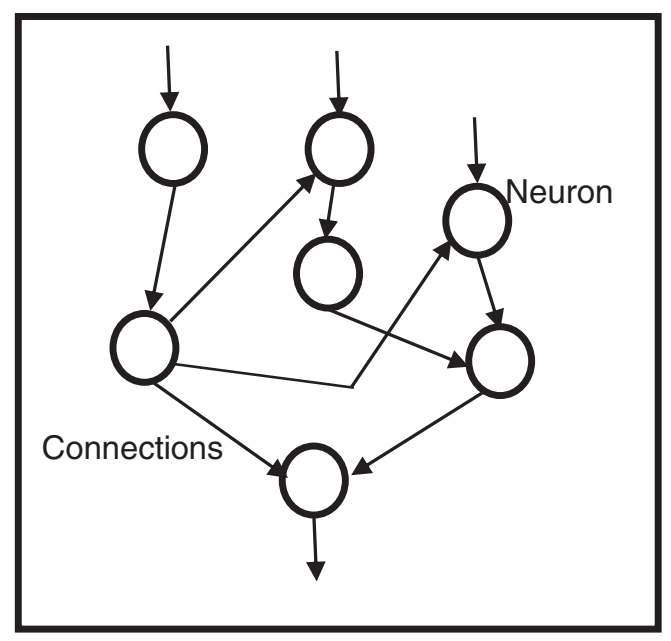

Figure 1. An example of a partial recurrent network.

\section{Related work}

Various techniques for extracting features from images have been proposed in literature. Rameshbabudurai et al (2012) proposed feature extraction from images using Discrete Cosine Transform (DCT). Gaussian Fuzzy Feed Forward Neural Network (NN) was used for classification of the extracted features. Neural Network was improved by optimizing momentum and learning rate through GA. Jeyabharathi (2013) analyzed feature extraction techniques including Principal Component Analysis (PCA), Linear Discriminant Analysis, and Independent Component Analysis (ICA) and measured performance using Support Vector Machine (SVM) and Neural Network (NN). Based on performance of Recognition Rate and F-Score, it was seen that PCA with SVM provides better recognition accuracy than other techniques investigated. Gabor wavelets based features were extracted from medical mammogram images representing normal tissues or benign/malignant tumors by Buciu \& Gacsadi (2009). When features were detected, PCA reduced data dimensionality. Alirezaie et al (1997) applied a Learning Vector Quantization ANN for multispectral supervised MR images classification. An image classification method based on NN model dealing with attentive objects tree structures was presented by $\mathrm{Fu}$ et al (2009). Other than regions provided by image segmentation, attentive objects extracted from a segmented image, by an attention-driven image interpretation algorithm, constructed the tree structure to represent an image. A framework for semi-supervised remote sensing image classification based on NNs with a flexible embedding regularizer to the loss function was presented by Ratle et al (2010).

A method using edge density histogram to extract medical images shape feature was proposed by Li-dong \& Yi-fei (2010). Then Euclidean distance and SVM were used for medical image retrieval and classification. A new classification-driven design scheme aimed at deriving wavelets that best represent between-class discrimination capability that spectral signatures convey through hyper spectral images was proposed by Daamouche \& Melgani (2009). This was attained through adoption of a polyphase representation of wavelet filter bank and formulating wavelet optimization issues within a PSO framework. Experiments showed that the new wavelet design outperformed Daubechies wavelets whatever be the classifier adopted for classification. 
Heuristic methods have been extensively used in CBIR for optimizing feature selection methods and improving efficiency of classifiers. Two kinds of two-stage feature selection schemes for weight optimization and descriptor subset selection were proposed by Zhao et al (2008). The proposed technique used Binary GA and a coded GA. Faria et al (2010) explored the idea of introducing algorithms that combine information from various descriptors. The new learning rank algorithms were based on three diverse learning techniques: Support Machines (CBIRSVM), Association Rules (CBIR-AR), and Genetic Programming (CBIR-GP). An ARD-FS system integrating Automatic Relevance Determination (ARD) and Feature Selection (FS) for Gaussian Process Classifier (GPC) based on PSO algorithm was proposed by Zhang et al (2012). The ARD-FS system reduces GPC complexity and simultaneously improved classification accuracy of hyper spectral images even with highly limited labeled samples and high-dimensional features.

An improved approach integrating GA and adaptive PSO with feed forward NNs for image compression was proposed by Feng et al (2011). The hybrid GA with a new mutation strategy and PSO trained the NN to near global optimum weights and thresholds first. The results showed that at the same compression rate application of optimized NN in image compression achieved better image quality when compared to traditional NN application. A user-oriented CBIR mechanism based on Interactive Genetic Algorithm (IGA) was proposed by Lai \& Chen (2011). Color attributes like mean value, standard deviation, and color image bitmap were used as features for retrieval. To reduce the gap between retrieval results and users' expectation, IGA was used to help users identify images which satisfied users' need. A new, multilayer NN based on Fast Bacterial Swarming Algorithm (FBSA) for image compression was presented by Chu et al (2008). The proposed technique combined the traditional structure of a two-layer symmetric ANN and FBSA, proved quick with good performance for high-dimensional function optimization problems. Comparison of reconstructed images qualities showed effective improvement using the new algorithm than using conventional Back Propagation network.

From literature survey it can be seen that Neural Network has been effectively used for compression and image retrieval problems. However, compressed medical image retrieval has not been studied extensively in the literature. In this paper, investigation has been carried out to retrieve image similar to query medical image which is compressed for efficient storage and transmission.

\section{Methodology}

The block diagram of the proposed method is shown in figure 2. Images are first compressed using the simple Haar wavelet (Lewis \& Knowles 1992) to obtain the compressed image that will be used to obtain the feature space. Texture features are extracted using Gabor filter and shape features are extracted using Sobel edge detector. The features are concatenated and classified using Partial Recurrent Neural Network (PRNN) (Haykin 2004). The parameters of PRNN are optimized using the proposed PSO-CS heuristic.

\subsection{Image compression}

Haar Transform is applied to the medical images to shift from the space domain to the local frequency domain (Talukder \& Harada 2007). User queries contain large constant-colored regions which are well represented by Haar transforms. A drawback of Haar based lossy compression is the tendency to produce blocky image artifacts at high compression rates. 


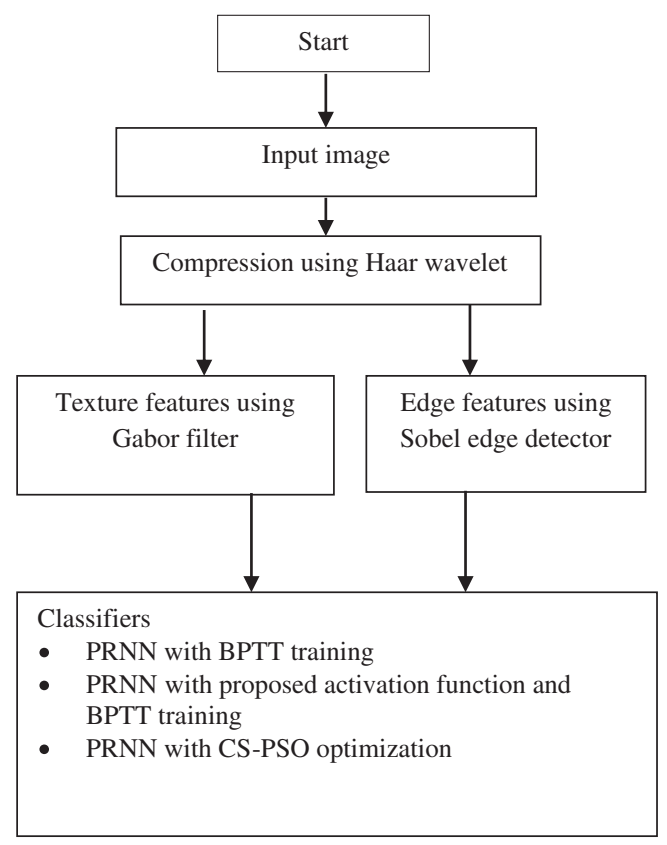

Figure 2. Flowchart of proposed methodology.

Family of $N$ Haar functions $h_{k}(t)$ are defined on interval $0 \leq t \leq 1$ (Mulcahy 1997). The shape of Haar function, of index $k$, is determined by two parameters: $p$ and $q$, where

$$
k=2^{p}+q-1
$$

where $k$ is in the range of $k=0,1,2, \cdots, N-1$

When $k=0$, the Haar function is defined as a constant $h_{0}(t)=1 / \sqrt{N}$ when $k>0$, Haar function is defined as

$$
h_{k}(\mathrm{t})=\frac{1}{\sqrt{N}}\left\{\begin{array}{cc}
2^{p / 2} & (q-1) / 2^{p} \leq t<(q-0.5) / 2^{p} \\
-2^{p / 2} & (q-0.5) / 2^{p} \leq t<q / 2^{p} \\
0 & \text { otherwise }
\end{array} .\right.
$$

From Haar function it is seen that $p$ determines amplitude and width of the function's nonzero part, while $q$ determines the position of non-zero part of Haar function (Morton \& Petersen 1997).

Discrete Haar functions are the basis of Haar matrix $\mathbf{H}$

$$
\begin{gathered}
H_{2 N}=\left[\begin{array}{c}
H_{N} \otimes[1,1] \\
I_{N} \otimes[1,-1]
\end{array}\right] \\
\mathrm{H}(0)=1
\end{gathered}
$$




$$
\text { where } \mathbf{I}_{N}=\left[\begin{array}{ccccc}
1 & 0 & \cdots & 0 & 0 \\
0 & 1 & \cdots & 0 & 0 \\
\vdots & \vdots & \ddots & \vdots & \vdots \\
0 & 0 & \cdots & 1 & 0 \\
0 & 0 & \cdots & 0 & 1
\end{array}\right]
$$

and $\otimes$ is the Kronecker product.

The Haar matrix is real and orthogonal and hence

$$
\begin{gathered}
\mathbf{H}=\mathbf{H}^{*} \\
\mathbf{H}^{-1}=\mathbf{H}^{T} .
\end{gathered}
$$

\subsection{Feature extraction}

For texture features, Gabor filters serve as excellent band-pass filters for uni-dimensional signals. The complex Gabor filter real/imaginary components are phase sensitive, i.e., their response to a sinusoid is another sinusoid. It is useful to compute overall output of two out of phase filters. A common way to do so is adding squared output (energy) of a filter, equally to get the magnitude corresponding to magnitude of complex Gabor filter output (Movellan 2009).

Multiplication of cosine/sine (even/odd) wave with Gaussian windows is defined as a onedimensional Gabor filter and given by (Prasad \& Domke 2005),

$$
\begin{aligned}
& g_{e}(x)=\frac{1}{\sqrt{2 \pi \sigma}} e^{\frac{x^{2}}{e^{2 \sigma^{2}}}} \cos \left(2 \pi w_{o} x\right) \\
& g_{o}(x)=\frac{1}{\sqrt{2 \pi \sigma}} e^{\frac{x^{2}}{e^{2 \sigma^{2}}}} \sin \left(2 \pi w_{o} x\right) .
\end{aligned}
$$

A Gabor filter is got by modulating a sinusoid with Gaussian. For One Dimensional (1D) signals, 1D sinusoid is modulated with Gaussian which responds to some frequency, at a localized portion of the signal. Let $\mathrm{g}(x, y, \theta, \varphi)$ be function defining a Gabor filter centered at the origin with $\theta$ as spatial frequency and $\varphi$ as orientation. Gabor filter can be defined as

$$
g(x, y, \theta, \phi)=\exp \left(-\frac{x^{2}+y^{2}}{\sigma^{2}}\right) \exp (2 \pi \theta i(x \cos \phi+y \sin \varphi)) .
$$

It is shown that $\sigma$, standard deviation of Gaussian kernel depends on spatial frequency to measured.

Sobel operator is used for edge detection algorithms. It is, technically, a discrete differentiation operator, computing image intensity functions. At each image point, the Sobel operator's result is either corresponding opposite of the gradient vector or the vector's norm. The Sobel operator convolves the image with a separable integer valued filter in horizontal/vertical directions and so is inexpensive regarding computations (Jansi \& Subashini 2012).

In theory, the operator includes a pair of $3 \times 3$ convolution masks shown in figure 3 . One mask is the other rotated by $90^{\circ}$. Figure 3 shows the convoluted mask in the counter clock wise direction by 90 degrees.

These masks respond maximally to edges running vertically/horizontally relative to the pixel grid, one mask for each of two perpendicular orientations. Masks can also be applied separately 


\begin{tabular}{|l|l|l|}
\hline-1 & 0 & +1 \\
\hline-2 & 0 & +2 \\
\hline-1 & 0 & +1 \\
\hline
\end{tabular}

$\mathrm{G}_{\mathrm{X}}$

\begin{tabular}{|l|l|l|}
\hline+1 & +2 & +1 \\
\hline 0 & 0 & 0 \\
\hline-1 & -2 & -1 \\
\hline
\end{tabular}

$\mathrm{G}_{\mathrm{Y}}$

Figure 3. Sobel convolution masks.

to the input image, producing separate gradient component measurements in each orientation (called $\mathrm{G}_{\mathrm{x}} / \mathrm{G}_{\mathrm{y}}$ ). These combine to find the gradient's absolute magnitude at each point and the gradient's orientation. The gradient magnitude is given by (Matthews 2002):

$$
|G|=\sqrt{G_{x}^{2}+G_{y}^{2}}
$$

\subsection{Partial recurrent neural network classifier}

In simple PRNN some nodes form part of a feedforward structure, others provide sequential context and receive feedback from other nodes. Context unit weights are processed similar to those for input units using backpropagation. Context units receive time-delayed feedback from second layer units. Training data includes inputs and desired successor outputs.

In the proposed PRNN, the descent of the sigmoidal function used in the hidden layer is dynamically changed during the training of the network. The sigmoidal activation function is given as

$$
\frac{1}{\left(1+\exp ^{-x^{*} n e t}\right)}
$$

where the steepness parameter $x=1$ in most cases and execution of gradient descent on the steepness improves the BP algorithm. The value of $x$ is computed based on the system error $E$, with respect to $x$,

$$
x_{\text {new }}=x_{\text {old }}-\varepsilon^{*}\left(\frac{-\partial E}{\partial x_{\text {old }}}\right)
$$

where $\varepsilon$ is a small positive integer and is assigned a value of 1.5 in this work.

$$
\begin{gathered}
\frac{\partial E}{\partial x_{\text {old }}}=\frac{\partial E}{\partial f_{\text {out }}} * \frac{\partial f_{\text {out }}}{\partial x_{\text {old }}} \\
\frac{\partial f_{\text {out }}}{\partial x_{\text {old }}}=\left(\frac{\exp ^{x_{\text {old }} * i n p}}{1+\exp ^{x_{\text {old } * i n p}}}\right)^{2} * \text { inp } \\
E=\frac{1}{N_{p}} \sum_{k=1}^{N_{p}}\left(\text { aout }_{k}-\text { fout }_{k}\right)^{2}
\end{gathered}
$$


where the system error $E$, for all the patterns $(N p)$ is calculated as the sum of the squared error for each pattern. In this work the Backpropagation Through Time (BPTT) algorithm which is an extension of the Back Propagation algorithm used in standard neural networks is used for training the network. BPTT performs gradient descent on the complete unfolded network. For a time interval between $t_{0}$ and $t_{1}$, the cost function can be computed as the sum of the standard error at each time step. The total error is given by

$$
\begin{aligned}
& E_{\text {tot }}\left(t_{0}, t_{1}\right)=\sum_{t=t_{0}}^{t_{1}} E_{S} \\
& \text { where } \\
& E_{\text {tot }}=\text { total error } \\
& E_{S}=\text { Sum of error function at time } t
\end{aligned}
$$

For each time step the gradient descent weight update is given by

$$
\Delta w_{i j}=-\eta \frac{\partial E_{t o t}\left(t_{0}, t_{1}\right)}{\partial w_{i j}}
$$

$\eta$ is the learning rate and can have value between 0.001 and 1. Small value of learning rate increases the convergence time. This work uses a novel Cuckoo Search (CS) for finding the ideal learning rate. The parameters of CS plays a crucial role in finding the optimal solution. Cuckoo Search algorithm performance highly depends on the set parameter values and finding the optimal value needs knowledge of the problem statement. In this work, the optimization of the parameters is seen as a meta-optimization problem and Particle Swarm Optimization is used to find the optimal CS parameters. In effect for finding the optimal value of the learning rate of BPTT a parameter optimized CS is used.

CS is a heuristic search algorithm proposed by Yang \& Deb (2009) inspired by reproduction strategy of cuckoos. At the basic level, cuckoos lay eggs in other host birds' nests of different species. The host bird might feel that the eggs are not its and either destroy egg or abandon nest totally. Three idealized rules for Cuckoo Search are:

1. Each cuckoo lays one egg and puts it in a randomly chosen nest;

2. Best nests with high quality eggs carry over to next generations;

3. Available host nests number is fixed and egg laid by a cuckoo is discovered by host bird with probability pa $\in[0,1]$.

The pseudo-code for CS algorithm is given (Tuba et al 2011):

\section{Start}

Objective function $f(x), x=\left(x_{1}, x_{2} \ldots x_{u}\right)^{T}$

Generating initial population of $n$ host nests $x_{i}(i=1,2, \ldots n)$

While ( $t<$ MaxGenerations) and (!termin.condit.)

Move a cuckoo randomly via Lévy flights

Evaluate its fitness $\mathrm{Fi}$

Randomly choose nest $j$ among $n$ available nests

If $(F i>F j)$ Replace $j$ by the new solution;

Fraction pd of worse nests are abandoned andnew nests are being built; 
Keep the best solutions or nests with quality solutions;

End while

Rank the solutions and find the current best

Post process and results

End

For generating new solutions, Levy flight is used to and given by

$$
x_{i}(t+1)=x_{i}(t)+\alpha \wedge \operatorname{Levy}(\lambda)
$$

where $\alpha$ is step size and $\wedge$ is entry-wise multiplication. The Levy flight step lengths are distributed by

$$
\text { Levy } u=t^{-\lambda}, 1<\lambda \leq 3
$$

In the proposed hybrid optimization, the CS makes use of PSO to achieve better solutions. The parameters $p_{d}, \lambda$ and $\alpha$ in the CS help the algorithm to find global and local solutions, respectively. The parameters $p_{d}$ and $\alpha$ are used in adjusting convergence rate of algorithm. The traditional CS algorithm uses fixed value for both $p_{d}$ and $\alpha$. If the value of $p_{d}$ is small and the value of $\alpha$ is large, poor performance is achieved due to considerable increase in number of iterations. If the value of $p_{d}$ is large and the value of $\alpha$ is small, the speed of convergence is high leading to poor solutions (Valian et al 2011). In this work, PSO is used to find optimal value of $p_{d}$ and $\alpha$. Pseudo-code of PSO algorithm is given by (Settles 2005):

PSO is inspired by fish and bird swarm intelligence while Cuckoo Search (CS) is based on breeding behavior and brood parasitism of specific cuckoo species (Zheng \& Zhou 2012). Such nature-inspired meta-heuristic algorithms have been extensively used in various optimization problems, including NP-hard and NP - complete problems. The success of a population-based algorithms is dependent on proper trade-off between exploration and exploitation. A poor balance between exploration and exploitation results in a weak optimization method suffering from premature convergence and/or trapping in a local optima.

For each particle

Initialize particle

End For

Do

For each particle

Calculate fitness value of the particle $f(p)$

* updating particle's best fitness value so far* /

If $f(p)$ is better than pbest

set current value as the new pbest

End For

/* updating population's best fitness value so far)*/

Set gbest to the best fitness value of all particles

For each particle

Calculate particle velocity according equation

$v_{i}^{d}=w v_{i}^{d}+c_{1} r_{1}\left(p_{i}^{d}-x_{i}^{d}\right)+c_{2} r_{2}\left(p_{g}^{d}-x_{i}^{d}\right)$

Update particle position according equation

$x_{i}^{d}=x_{i}^{d}+v_{i}^{d}$

End For

While maximum iterations OR minimum error criteria is not attained 
Table 1. Parameters used in the proposed model.

\begin{tabular}{lc}
\hline Gabor filter parameters & $15,45,75$ degree \\
Orientation $\theta$ & 0 degree \\
Phase offset values used $\phi$ & \\
Neural Network parameters & 50 \\
Input neuron & 1 \\
Number of hidden layer & 10 \\
Number of neurons in hidden layer & Sigmoidal, proposed \\
Activation function of hidden layer & BPTT, proposed \\
Training algorithm & $<0.01$ or 500 iterations \\
Termination criteria & \\
Particle swarm optimization parameters & 1 \\
w 1 & 0.5 \\
c1 2 & 0.5 \\
Rand & {$[0.1]$} \\
Number of iterations & 500 \\
Cuckoo search parameters & \\
Levy step size $\alpha$ & Between 0 and 1.5 \\
$\lambda$ & Between 1 and 3 \\
\hline
\end{tabular}

The design parameters used in this work are given in table 1.

A sample medical images used in the experimental setup is shown in figure 4 .

Using the real part of the Gabor filter kernel at different offset of 15, 45 and 75 degree texture features were extracted from the compressed images. The min, max, average and variance of the filtered images and the edges obtained from Sobel edge detector are concatenated to form the feature space. The Gabor output for an image is shown below in table 2 and figure 5 shows the three dimensional distribution plot.

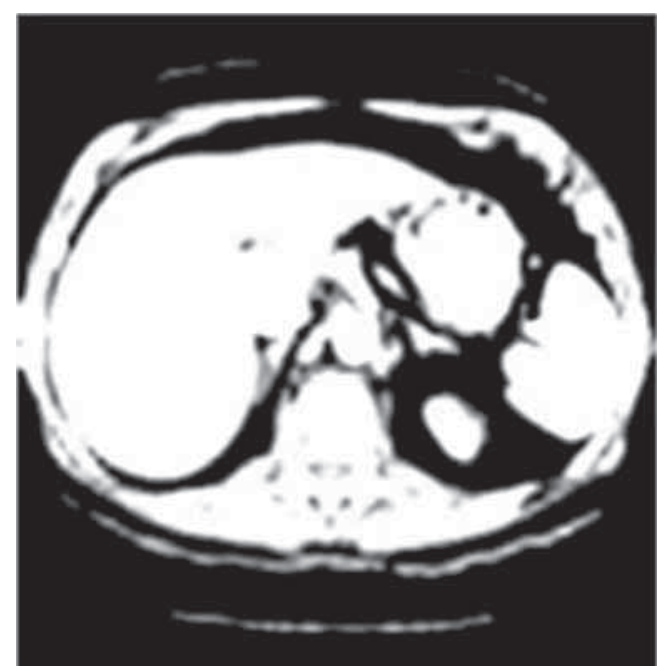

Figure 4. Images used in the investigation. 
Table 2. Gabor output for an image.

\begin{tabular}{cccccccccc}
\hline 110.51 & 611.48 & 1055.8 & 1044.4 & 631.1 & 33.017 & 468.66 & 686.46 & 642.5 & 407.61 \\
276.05 & 126.67 & 472.3 & 613.83 & 521.86 & 276.98 & 32.571 & 51.731 & 34.951 & 0 \\
324.21 & 216.69 & 12.162 & 214.72 & 386.42 & 434.38 & 359.34 & 206.33 & 111.59 & 45.507 \\
91.416 & 347.83 & 636.16 & 601.18 & 226.65 & 318.38 & 763.66 & 904.29 & 673.98 & 199.88 \\
456.33 & 191.83 & 194.76 & 535.09 & 669.28 & 527.26 & 157.07 & 260.58 & 534.49 & 559.79 \\
237.33 & 203.74 & 554.09 & 641.21 & 456.7 & 100.05 & 270.14 & 479.52 & 455.99 & 266.61 \\
257.58 & 220.76 & 89.097 & 78.42 & 192.16 & 182.46 & 57.513 & 123.71 & 256.84 & 295.18 \\
471.36 & 477.86 & 257.53 & 62.029 & 282.63 & 264.13 & 9.8689 & 277.49 & 442.95 & 406.35 \\
375.99 & 512.75 & 422.62 & 163.1 & 150.74 & 401.09 & 541.9 & 523.15 & 375.18 & 200.91 \\
413.48 & 129.32 & 340.29 & 749.73 & 911.14 & 757.03 & 495.23 & 181.74 & 85.337 & 217.38 \\
\hline
\end{tabular}

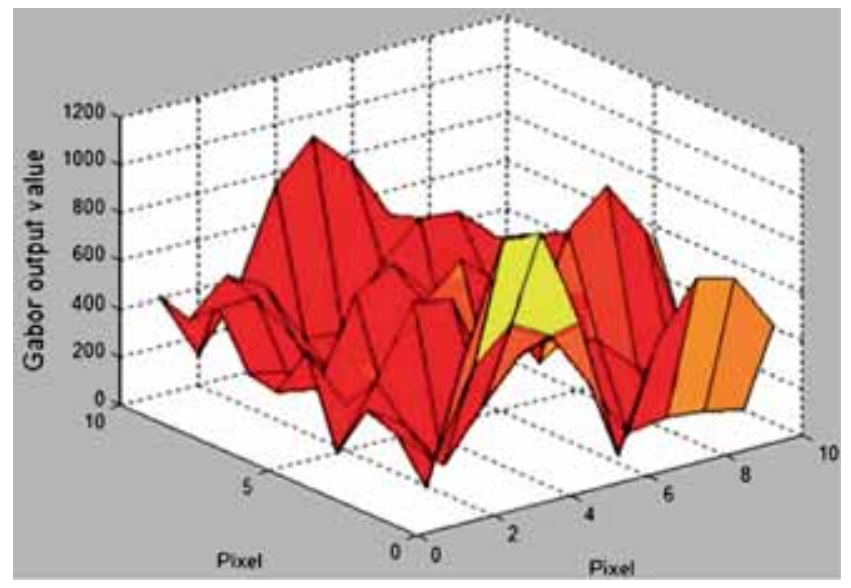

Figure 5. 3-D output of the Gabor output values.

\section{Experimental results}

Six thousand raw medical images were obtained from National Biomedical Imaging Archive (https://imaging.nci.nih.gov/ncia) and compressed using Haar Wavelet. Edge and texture features were extracted using Sobel edge detector and Gabor filters respectively. Features are ranked using Mutual Information and the top 50 features are selected. Experiments were run for 10-fold cross validation using PRNN classifier with proposed activation function and learning parameter optimization.

Figure 6 shows the average classification accuracy after 5 runs.

It is observed that the proposed activation RNN-BPTT with CS-PSO optimization improves classification accuracy by $2.17 \%$ compared to the method where CS parameters are not optimized. Classification accuracy improves by $8.97 \%$ when the learning parameter is not optimized and a learning rate of 0.01 is selected.

The proposed activation function improves the classification accuracy by $2.31 \%$ compared to sigmoidal activation function after learning rate is optimized using CS-PSO algorithm. The precision, recall and $\mathrm{F}$ measure obtained are shown in table 3. C1, C2, C3, C4 and C5 represent the class labels of the image under test. 


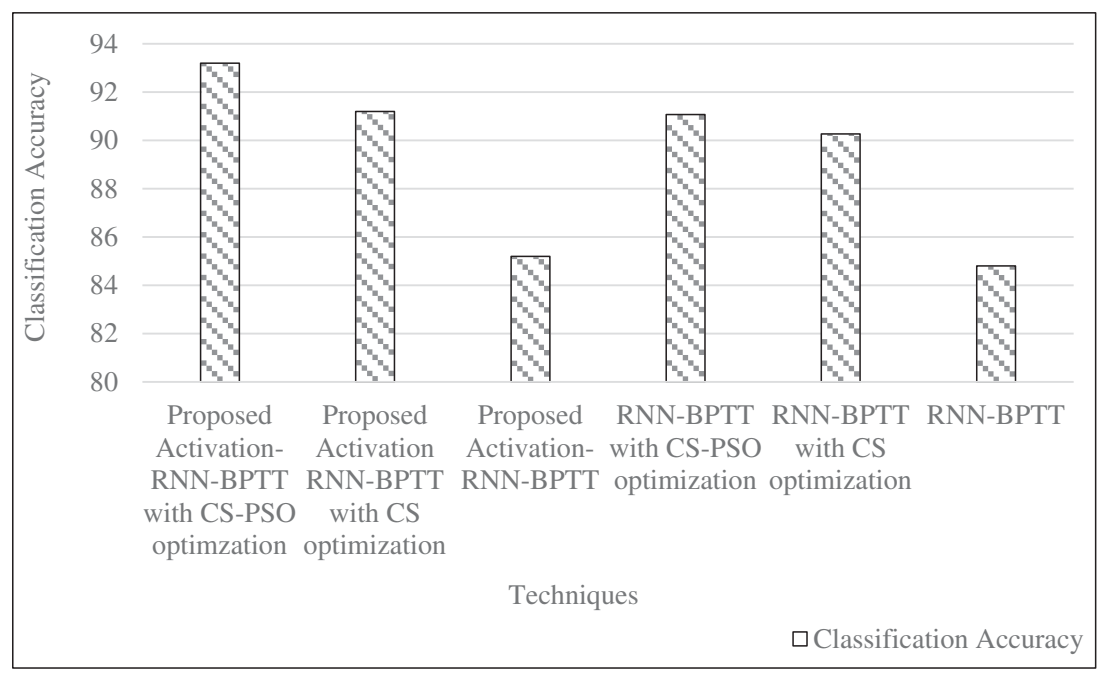

Figure 6. Classification accuracy for a larger dataset.

Table 3. Average precision, recall and F Measure obtained for all the experiments.

\begin{tabular}{|c|c|c|c|c|c|c|c|}
\hline Technique used & Precision & Recall & F measure & Technique used & Precision & Recall. & F measure \\
\hline Proposed & & & & RNN-BPTT with & & & \\
\hline Activation- RNN- & & & & CS-PSO optimization & & & \\
\hline $\begin{array}{l}\text { BPTT with CS- } \\
\text { PSO optimization }\end{array}$ & & & & & & & \\
\hline $\mathrm{C} 1$ & 0.9467 & 0.9404 & 0.9435 & $\mathrm{C} 1$ & 0.9267 & 0.9267 & 0.9267 \\
\hline $\mathrm{C} 2$ & 0.9267 & 0.9456 & 0.9361 & $\mathrm{C} 2$ & 0.9133 & 0.8896 & 0.9013 \\
\hline $\mathrm{C} 3$ & 0.92 & 0.92 & 0.92 & $\mathrm{C} 3$ & 0.9067 & 0.9007 & 0.9037 \\
\hline $\mathrm{C} 4$ & 0.9267 & 0.9586 & 0.9424 & $\mathrm{C} 4$ & 0.9133 & 0.9514 & 0.932 \\
\hline $\mathrm{C} 5$ & 0.94 & 0.8981 & 0.9186 & C5 & 0.8933 & 0.8874 & 0.8903 \\
\hline $\begin{array}{l}\text { Proposed } \\
\text { Activation RNN- } \\
\text { BPTT with CS } \\
\text { optimization }\end{array}$ & & & & $\begin{array}{l}\text { RNN-BPTT with CS } \\
\text { optimization }\end{array}$ & & & \\
\hline $\mathrm{C} 1$ & 0.9267 & 0.9145 & 0.9206 & $\mathrm{C} 1$ & 0.9267 & 0.9267 & 0.9267 \\
\hline $\mathrm{C} 2$ & 0.9133 & 0.9257 & 0.9195 & $\mathrm{C} 2$ & 0.9133 & 0.8896 & 0.9013 \\
\hline $\mathrm{C} 3$ & 0.9067 & 0.8947 & 0.9007 & C3 & 0.9067 & 0.9007 & 0.9037 \\
\hline $\mathrm{C} 4$ & 0.8933 & 0.9371 & 0.9147 & $\mathrm{C} 4$ & 0.8733 & 0.9493 & 0.9097 \\
\hline $\mathrm{C} 5$ & 0.92 & 0.8903 & 0.9049 & C5 & 0.8933 & 0.8535 & 0.8729 \\
\hline $\begin{array}{l}\text { Proposed } \\
\text { Activation- RNN- } \\
\text { BPTT }\end{array}$ & & & & RNN-BPTT & & & \\
\hline $\mathrm{C} 1$ & 0.8533 & 0.8649 & 0.8591 & $\mathrm{C} 1$ & 0.84 & 0.8571 & 0.8485 \\
\hline $\mathrm{C} 2$ & 0.86 & 0.8377 & 0.8487 & $\mathrm{C} 2$ & 0.8533 & 0.8366 & 0.8449 \\
\hline C3 & 0.86 & 0.8543 & 0.8571 & $\mathrm{C} 3$ & 0.86 & 0.8487 & 0.8543 \\
\hline $\mathrm{C} 4$ & 0.8533 & 0.8477 & 0.8505 & $\mathrm{C} 4$ & 0.8533 & 0.8421 & 0.8477 \\
\hline C5 & 0.8333 & 0.8562 & 0.8446 & $\mathrm{C} 5$ & 0.8333 & 0.8562 & 0.8446 \\
\hline
\end{tabular}




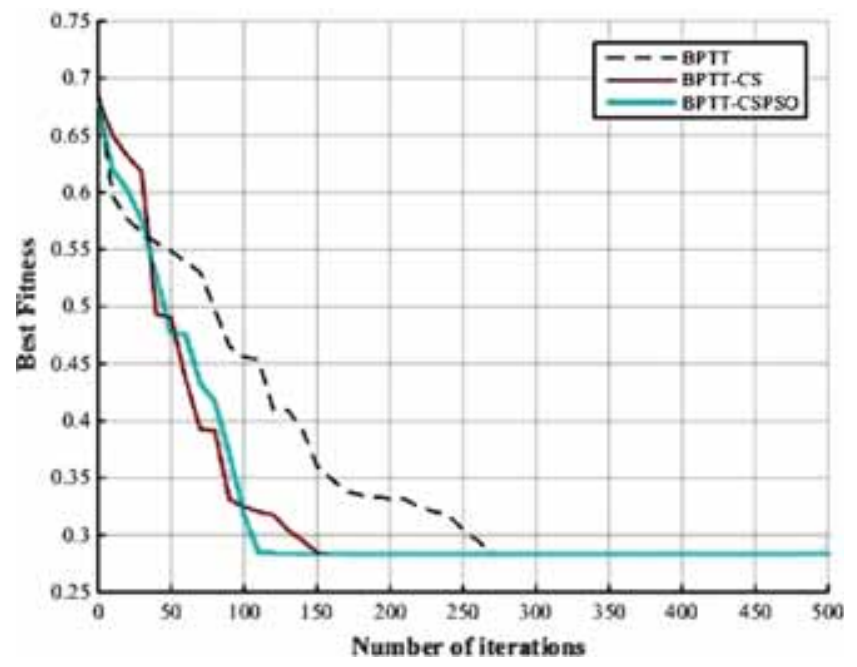

Figure 7. Convergence characteristics of the training algorithm.

It can be observed from results that false positives and false negatives are almost equal giving to precision and recall values which are almost same. This characteristic is desirable for the medical image classification system.

The convergence characteristics of the Neural Network under different scenarios are shown in figure 7 .

From figure 7 it can be observed that optimization of learning rate improves the convergence rate of the neural network with CS based optimization converging at $149^{\text {th }}$ iteration and the proposed CS-PSO technique algorithm converging at $114^{\text {th }}$ iteration and hence significantly decreasing the training time.

\section{Conclusion}

With development and availability of large number of medical image capturing devices like CT, MRI and C-Arm, digital image collection size has increased and mostly stored in compressed form. This work investigated classification algorithms for the image retrieval problem. Edge and texture features were extracted and the top ranked features are selected using Mutual Information. The extracted features are classified using Partial Recurrent Neural Network (PRNN) with a novel activation function. The learning rate of the neural network is optimized using Cuckoo Search and the proposed Meta Parameter Optimization of CS parameters using Particle Swarm Optimization. Experimental results show improved performance. Using 6000 medical images consisting of five classes, a classification accuracy of $93.2 \%$ was obtained. The proposed techniques not only improved the classification accuracy but also ensured faster learning by the proposed neural network methodology.

\section{References}

Alirezaie J, Jernigan M E and Nahmias C 1997 Neural network-based segmentation of magnetic resonance images of the brain. IEEE Trans. Nuclear Sci. 44(2): 194-198 
Barrett S 2007 Content-based image retrieval: a short term and long-term learning approach. http://digital. cs.usu.edu/ xqi/Teaching/REU07/Website/Samuel/SamFinalPaper.pdf

Buciu I and Gacsadi A 2009 Gabor wavelet based features for medical image analysis and classification. In: Second International Symposium on Applied Sciences in Biomedical and Communication Technologies, 2009. ISABEL 2009, IEEE. pp 1-4

Chu Y, Mi H, Ji Z and Shao Z B 2008 Image compression using multilayer neural networks based on Fast Bacterial Swarming Algorithm. In: 2008 International Conference on Machine Learning and Cybernetics, IEEE vol 5, pp 2890-2893

Daamouche A and Melgani F 2009 Swarm intelligence approach to wavelet design for hyperspectral image classification. Geosci. Remote Sensing Lett. IEEE 6(4): 825-829

Faria F F, Veloso A, Almeida H M, Valle E, Torres R D S, Gonçalves M A and Meira Jr W 2010 Learning to rank for content-based image retrieval. In: Proceedings of the international conference on Multimedia information retrieval, ACM. pp 285-294

Feng H, Tang M and Qi J 2011 A back-propagation neural network based on a hybrid genetic algorithm and particle swarm optimization for image compression. In: 2011 4th International Congress on Image and Signal Processing (CISP), IEEE vol 3, pp 1315-1318

Fu H, Zhang S, Chi Z, Feng D D and Zhao X 2009 Tree structures with attentive objects for image classification using a neural network. In: International Joint Conference on Neural Networks, 2009. IJCNN 2009, IEEE pp 898-902

Graves A, Fernández S and Schmidhuber J 2007 Multi-dimensional recurrent neural networks. In: Artificial Neural Networks-ICANN 2007 pp 549-558. Springer Berlin Heidelberg

Haralick Robert M, Shanmugam Karthikeyan and Dinstein Its'Hak 1973 Textural features for image classification. IEEE Trans. Syst. Man Cybern. 6(1973): 610-621

Haykin Simon 2004 Neural networks: A comprehensive foundation 2: 2004

Jansi S and Subashini P 2012 Optimized adaptive thresholding based edge detection method for MRI brain images. Int. J. Comput. Appl. 51(20): 1-8

Jeyabharathi D 2013 Performance analysis of feature extraction and classification techniques in CBIR. In: 2013 International Conference on Circuits, Power and Computing Technologies (ICCPCT), 20-21 March 2013

Lai C C and Chen Y C 2011 A user-oriented image retrieval system based on interactive genetic algorithm. IEEE Trans. Instrum. Measurement 60(10): 3318-3325

Lewis Adrian S and Knowles G 1992 Image compression using the 2-D wavelet transform. IEEE Trans. Image Process. 1(2): 244-250

Li-dong F and Yi-fei Z 2010 Medical image retrieval and classification based on morphological shape feature. In: 2010 3rd International Conference on Intelligent Networks and Intelligent Systems (ICINIS), IEEE pp 116-119

Matthews J 2002 An introduction to edge detection: The Sobel edge detector. Available at http://www. generation5.org/content/2002/im01.asp

Morton P and Petersen A 1997 Image compression using the Haar wavelet transform. College of the Redwoods

Movellan J R 2009 Tutorial on Gabor Filters [M/OL]. http://mplab.ucsd.edu/tutorials/gabor.pdf

Mulcahy C 1997 Image compression using the Haar wavelet transforms. Spelman Sci. Math. J. 1(1): 22-31

Prasad V S N and Domke J 2005 Gabor filter visualization. Technical Report, University of Maryland

Rajaei A and Rangarajan L 2011 Wavelet features extraction for medical image classification. Res. Cell: Int. J. Eng. Sci. 4: 131-141. ISSN: 2229-6913 Issue Sept 2011

Rameshbabudurai C, Duraisamy V and Vinothkumar C 2012 Improved content based image retrieval using neural network optimization with genetic algorithm. Int. J. Emerging Technol. Adv. Eng. 2(7). ISSN 2250-2459

Ratle F, Camps-Valls G and Weston J 2010 Semi supervised neural networks for efficient hyperspectral image classification. IEEE Trans. Geosci. Remote Sensing 48(5): 2271-2282

Schaefer G 2010 Content-based retrieval of compressed images. In: DATESO, pp 175-185 
Settles M 2005 An introduction to particle swarm optimization. Department of Computer Science, University of Idaho

Smeulders Arnold W M et al 2000 Content-based image retrieval at the end of the early years. IEEE Trans. Pattern Anal. Mach. Intell. 22(12): 1349-1380

Talukder K H and Harada K 2007 Haar wavelet based approach for image compression and quality assessment of compressed image. In: IAENG Int. J. Appl. Math. pp 49-56

Tuba M, Subotic M and Stanarevic N 2011 Modified cuckoo search algorithm for unconstrained optimization problems. In: Proceedings of the 5th European conference on European computing conference, pp 263-268. World Scientific and Engineering Academy and Society (WSEAS)

Vadapalli H B, Nyongesa H and Omlin C W P 2010 Recurrent neural networks for facial action unit recognition from image sequences. In: Proceedings of the 2009 International Conference on Image Processing, Computer Vision, \& Pattern Recognition, IPCV 2009, July 13-16, 2009, Las Vegas, Nevada, USA, 2 Volumes. CSREA Press 2009, ISBN 1-60132-119-8 pp 351-367

Valian E, Mohanna S and Tavakoli S 2011 Improved cuckoo search algorithm for feedforward neural network training. Int. J. Artif. Intell. Appl. 2(3): 36-43

Virk I S and Maini R 2011 Content based image retrieval: Tools and techniques. Res. Cell: Int. J. Eng. Sci. 5: 21-35. ISSN: 2229-6913 Issue Dec. 2011

Wei Wei- Yi 2008 An introduction to image compression, National Taiwan University, Taipei, Taiwan, ROC

Yang X S and Deb S 2009 Cuckoo search via Lévy flights. In: World Congress on Nature and Biologically Inspired Computing, 2009. NaBIC 2009, IEEE. pp 210-214

Zhang X, Wang W, Li Y and Jiao L C 2012 PSO-based automatic relevance determination and feature selection system for hyperspectral image classification. Electron. Lett. 48(20): 1263-1265

Zhao T, Lu J, Zhang Y and Xiao Q 2008 Feature selection based on genetic algorithm for CBIR. In: Congress on image and signal processing, 2008. CISP'08, IEEE. vol 2, pp 495-499

Zheng H and Zhou Y 2012 A novel cuckoo search optimization algorithm based on Gauss distribution. J. Comput. Inf. Syst. 8: 4193-4200 